\title{
Performance of pfHRP2 versus pLDH antigen rapid diagnostic tests for the detection of Plasmodium falciparum: a systematic review and meta-analysis
}

Bo Li ${ }^{1}$, Zhiqiang Sun ${ }^{1}$, Xiaohan $\mathrm{Li}^{1,2}$, Xiaoxi Li ${ }^{1}$, Han Wang ${ }^{1}$, Weijiao Chen ${ }^{1}$, Peng Chen ${ }^{1}$, Mengran Qiao ${ }^{1}$, Yuanli Mao ${ }^{1}$

${ }^{1}$ Center for Clinical Laboratory, 302 Hospital of PLA, Beijing, China ${ }^{2}$ Graduate Student Team, Medical University of PLA, Beijing, China

Submitted: 29 December 2014

Accepted: 5 July 2015

Arch Med Sci 2017; 13, 3: 541-549

DOI: https://doi.org/10.5114/aoms.2017.67279

Copyright (C) 2017 Termedia \& Banach

\section{Abstract}

Introduction: There have been many inconsistent reports about the performance of histidine-rich protein 2 (HRP2) and lactate dehydrogenase (LDH) antigens as rapid diagnostic tests (RDTs) for the diagnosis of past Plasmodium falciparum infections. This meta-analysis was performed to determine the performance of pfHRP2 versus pLDH antigen RDTs in the detection of P. falciparum.

Material and methods: After a systematic review of related studies, Meta-DiSc 1.4 software was used to calculate the pooled sensitivity, specificity, positive likelihood ratio (PLR), negative likelihood ratio (NLR), and diagnostic odds ratio (DOR). Forest plots and summary receiver operating characteristic curve (SROC) analysis were used to summarize the overall test performance. Results: Fourteen studies which met the inclusion criteria were included in the meta-analysis. The summary performances for pfHRP2- and pLDH-based tests in the diagnosis of $P$. falciparum infections were as follows: pooled sensitivity, $96.3 \%(95.8-96.7 \%)$ vs. $82.6 \%$ (81.7-83.5\%); specificity, $86.1 \%$ (85.3-86.8\%) vs. $95.9 \%$ (95.4-96.3\%); diagnostic odds ratio (DOR), 243.31 (97.679-606.08) vs. 230.59 (114.98-462.42); and area under ROCs, 0.9822 versus 0.9849 (all $p<0.001$ ).

Conclusions: The two RDTs performed satisfactorily for the diagnosis of $P$. falciparum, but the pLDH tests had higher specificity, whereas the pfHRP2 tests had better sensitivity. The PfHRP2 tests had slightly greater accuracy compared to the pLDH tests. A combination of both antigens might be a more reliable approach for the diagnosis of malaria.

Key words: histidine-rich protein 2, lactate dehydrogenase, rapid diagnostic tests, Plasmodium falciparum.

\section{Introduction}

Malaria is an infectious disease with a worldwide distribution. According to the WHO, there are approximately 200 million cases of malaria and > 500000 deaths each year; most victims are children living in Africa. Five Plasmodium species are known to cause malaria in humans: $P$. falciparum, $P$. vivax, $P$. malariae, $P$. ovale, and $P$. knowlesi. $P$. falciparum is the most prevalent and pathogenic species; thus malaria due to $P$. falciparum is an important public health problem in both endemic and non-endemic settings [1].

\author{
Corresponding author: \\ Prof. Yuanli Mao \\ Center for Clinical Laboratory \\ 302 Hospital of PLA \\ Beijing, China \\ Phone: +861063879625 \\ Fax: +86 1063879625 \\ E-mail: pipi780816@aliyun. \\ com
}


A prompt and accurate laboratory diagnosis is the key to providing malaria patients with effective treatment. Currently, a microscopic examination is the main method by which to diagnose a malarial infection, but this method is time-consuming and requires an experienced microscopist, which can be impractical in remote areas. The polymerase chain reaction $(P C R)$ is a highly sensitive alternative to microscopy, but cost and lack of general availability limit the routine use of PCR in many health care settings. Currently, rapid diagnostic tests (RDTs) are more rapid and cost-effective methods by which to diagnose malarial infections; RDTs require minimum training and are widely used in many malaria-endemic areas. Most of the RDTs for Plasmodium species are based on the detection of histidine-rich protein 2 (HRP2) or parasite-specific lactate dehydrogenase (LDH) antigen. pfHRP2-based tests have shown good sensitivity in a variety of field settings; however, pfHRP2-based tests can only diagnose $P$. falciparum infections. Moreover, the proportion of false-positive results in these assays is high due to antigen persistence for up to several weeks after effective treatment. Unlike the pfHRP2-based tests, pLDH-based tests can detect all human-related Plasmodium species, and pLDH antigen is rapidly cleared from the blood after successful treatment [2-8]. In addition, pLDH tests have advantages over pfHRP2 tests; specifically, pLDH tests are not affected by the prozone effect or pfHRP2 gene deletions. The sensitivity of these tests has often been reported as lower than pfHRP2-based tests [9-11]. pLDH is a highly conserved RDT antigen, in contrast to pfHRP2, which is considered to be more variable due to the genetic diversity and deletion of the pfhrp2 gene. In the past, the deletion of the pfhrp2 gene was reported as a cause of false negative diagnoses for $P$. falciparum infections $[12,13]$.

Many studies have assessed the diagnostic accuracy of malaria RDTs; however, conflicting data have been reported on the performance of pfHRP2- and pLDH-based tests [14-17]. Therefore, we conducted a meta-analysis to evaluate the di- agnostic value of the two malaria RDTs. The objective of this review was to compare the accuracy of pfHRP2- and pLDH-based tests for the diagnosis of $P$. falciparum infection.

\section{Material and methods}

\section{Search strategy}

A comprehensive systematic search was performed for articles in databases, including PubMed, Embase, ISI Web of Science, and the Cochrane Library. No restriction was set with respect to the year of publication and language. To find possible missing articles, we searched the references of the included articles and relevant published articles manually. The search terms used for this study are listed in Table I.

\section{Inclusion and exclusion criteria}

The inclusion criteria for this study were as follows: (1) original research articles that directly compared the diagnostic performance of pfHRP2and pLDH-based immunochromatographic assays for the detection of $P$. falciparum; (2) microscopic examination of blood smears or a PCR-corrected microscopy method were used as the gold standard; and (3) sufficient data to construct $2 \times 2$ tables of test performance.

Exclusion criteria for this study were as follows: (1) repeat publications or more than one article using the same patient population (n.b., we only selected the most recent study for analysis); and (2) studies that were published as reviews, letters, case reports, editorials, or comments. Abstracts and titles of all possible studies for inclusion were assessed independently by two reviewers, and any disagreements were resolved by consensus.

\section{Selection of studies}

The title and abstract of all potentially relevant articles were read by two reviewers independently, and the full text of potentially eligible studies was

Table I. Search terms used in the systematic search for this study

\begin{tabular}{|ll|}
\hline Database & \multicolumn{1}{c|}{ Search terms } \\
\hline PubMed & $\begin{array}{l}\text { (Plasmodium falciparum [Mesh] OR falciparum malaria OR P. falciparum) AND (Histidine*rich } \\
\text { protein 2 OR Histidine*rich Protein II OR HRPII OR HRP2 OR PfHRP2 OR PfHRPII) AND (Lactate } \\
\text { dehydrogenase OR PfLDH OR Pf*pLDH) }\end{array}$ \\
\hline Embase & $\begin{array}{l}\text { 'Plasmodium falciparum' OR 'falciparum malaria' OR 'p. falciparum' AND ('histidine? rich } \\
\text { protein 2' OR 'histidine?rich protein ii' OR hrpii OR hrp2 OR pfhrp2 OR pfhrpii) AND (lactate } \\
\text { AND dehydrogenase OR pfldh OR pf?pldh) }\end{array}$ \\
\hline Web of Science & $\begin{array}{l}\text { (Plasmodium falciparum OR falciparum malaria OR P. falciparum) AND (Histidine*rich } \\
\text { protein 2 OR Histidine*rich Protein II OR HRPII OR HRP2 OR PfHRP2 OR PfHRPII) AND (Lactate } \\
\text { dehydrogenase OR PfLDH OR Pf*pLDH) }\end{array}$ \\
\hline The Cochrane Library & $\begin{array}{l}\text { (Plasmodium falciparum OR falciparum malaria OR P. falciparum) AND (Histidine-rich } \\
\text { protein 2 OR Histidine rich Protein II OR HRPII OR HRP2 OR PfHRP2 OR PfHRPII) AND (Lactate } \\
\text { dehydrogenase OR PfLDH OR PfpLDH) }\end{array}$ \\
\hline
\end{tabular}



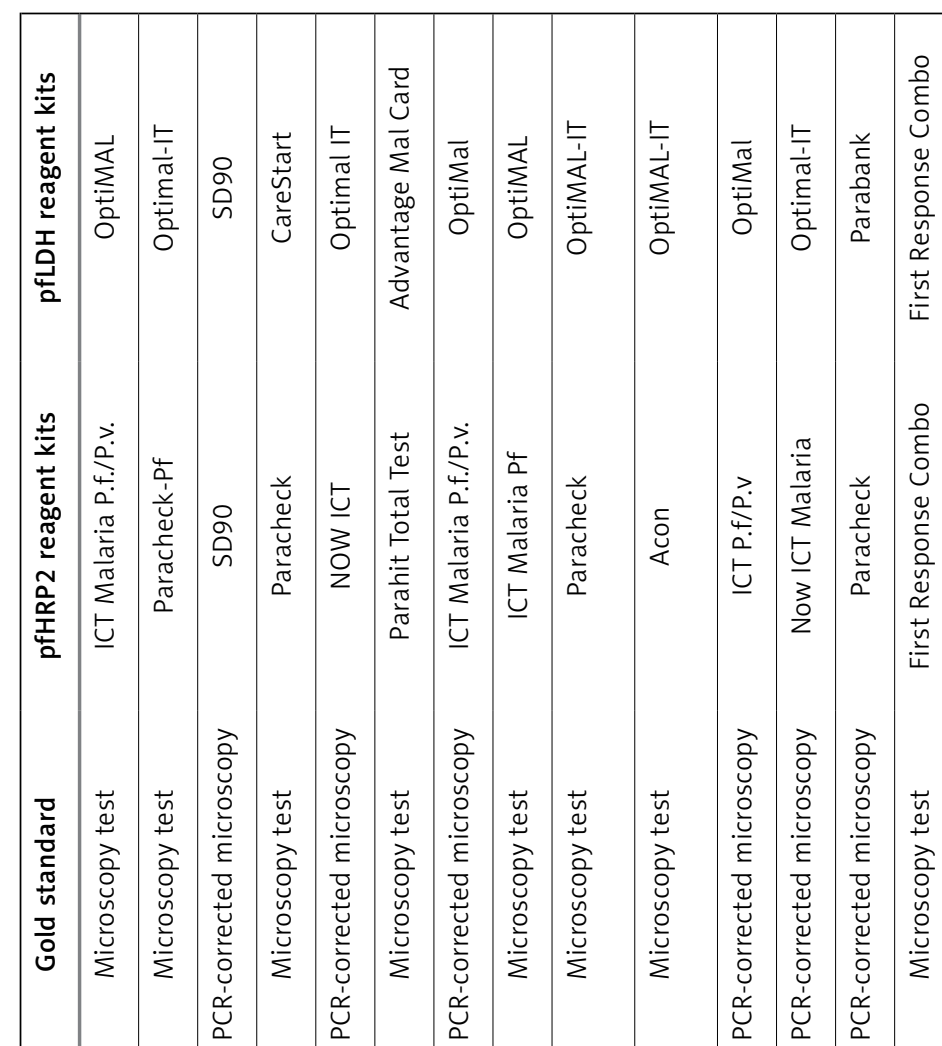

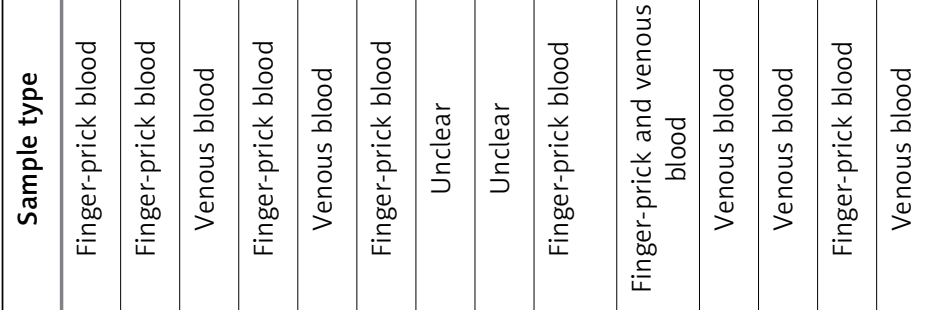

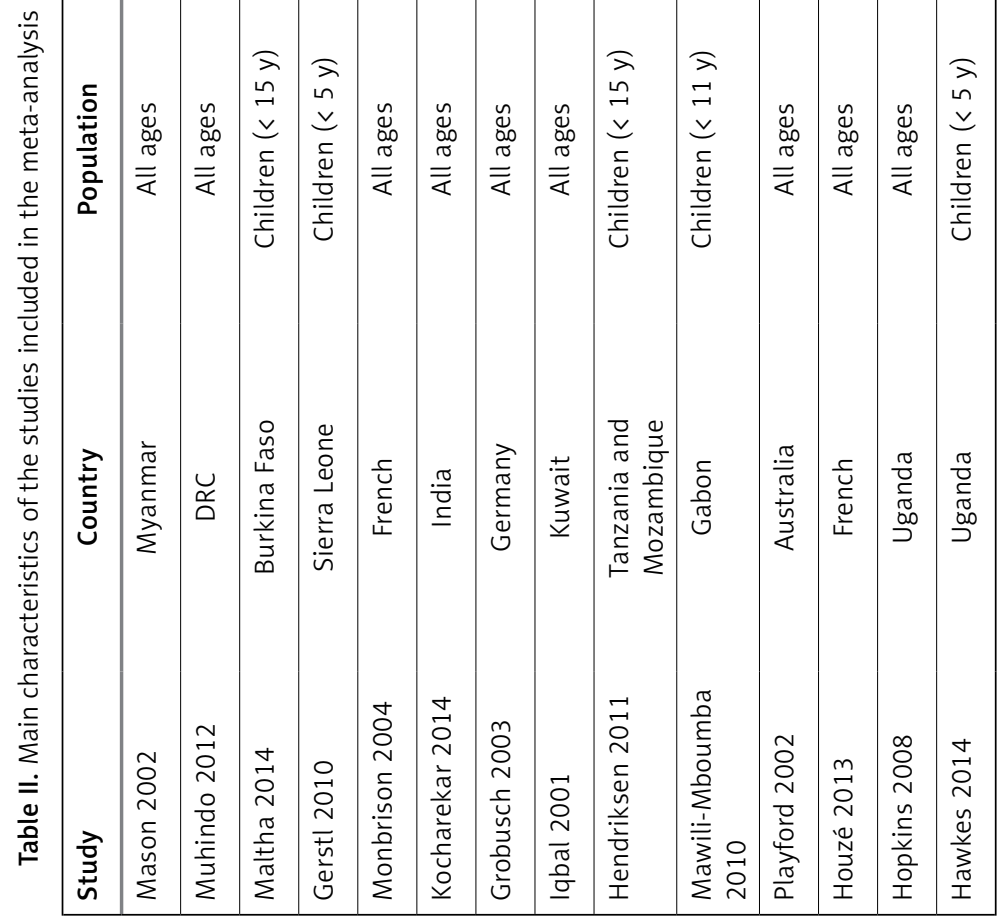


then retrieved for further assessment. Disagreements between the reviewers were resolved by consensus. The authors were contacted for further study details if necessary.

\section{Data extraction}

The following data were independently extracted from the included studies by two reviewers: authors; year of publication; geographical area; patient demographics; diagnostic criteria; test kits; and valid data for the analysis (the number of true-positive, false-negative, true-negative, and false-positive results).

\section{Assessment of study quality}

The Quality Assessment of Diagnostic Accuracy Studies (QUADAS-2) tool, as recommended by the Cochrane Collaboration, was used in our study to assess the quality of studies. The QUADAS-2 tool consists of four key domains that assess patient selection, index test, reference standard, and flow of patients through the study and timing of the in dex tests and reference standard. All of the included studies were evaluated as having low, high, or unclear risk of bias or concerns about applicability for each domain [18].

\section{Statistical analysis}

All statistical analyses were performed using Review Manager (RevMan) 5.2 and Meta-DiSc 1.4 software. Using RevMan 5.2, data were presented as forest plots and receiver operating characteristic curves (SROC). Meta-DiSc 1.4 software was used to calculate the pooled sensitivity, specificity, positive likelihood ratio (PLR), negative likelihood ratio (NLR), diagnostic odds ratio (DOR), and heterogeneity analysis. The threshold effect was

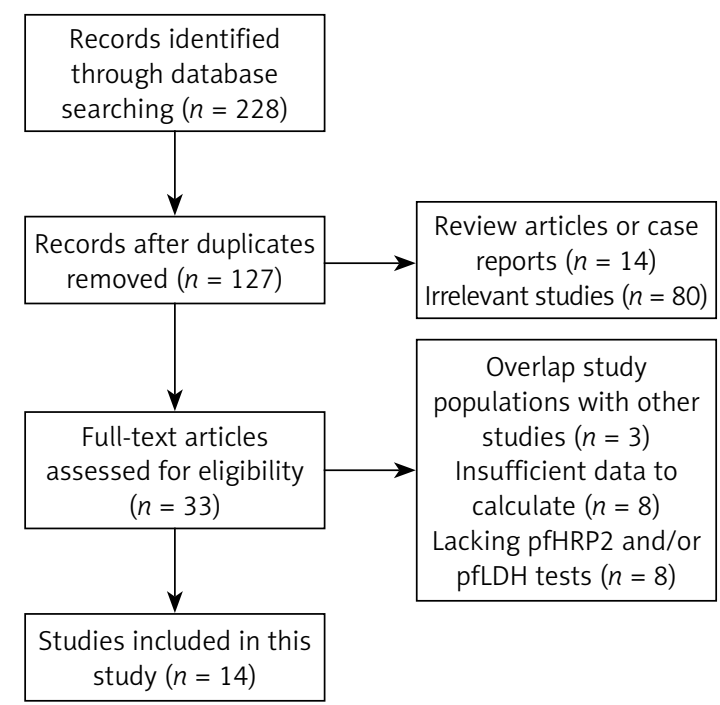

Figure 1 . Flow chart of the study selection process tested using the Spearman correlation coefficient. A $\chi^{2}$-based $Q$ statistical test was used to assess the heterogeneity induced by other factors $\left(I^{2} \leq\right.$ $50 \%$, insignificant heterogeneity; $R^{2}>50 \%$, significant heterogeneity). A random-effects model was used for analysis when the heterogeneity was apparent and a fixed-effects model was applied when insignificant heterogeneity existed. Meta-regression was performed to examine potential sources of heterogeneity. Subgroup analysis according to the source of heterogeneity was subsequently conducted [19].

\section{Results}

\section{Results of the search}

A total of 228 relevant articles were identified from the PubMed, ISI, EMBASE and Cochrane Library databases. One hundred one articles were excluded because of duplicate records. Ninety-four articles were excluded after reading the titles or abstracts and determining that the articles were reviews, case reports, or irrelevant studies. Thirty-three full text copies of potentially relevant studies were obtained from Internet databases. After full text reviews, 19 studies were excluded, as follows: 8 articles were excluded because they did not provide sufficient data to calculate the sensitivity and/or specificity; 8 articles were excluded because of lacking data of pfHRP2 and/or pLDH tests; and 3 articles were excluded because the articles had overlap study populations with other studies. Fourteen studies met all inclusion criteria and were entered into the meta-analysis, including 15,909 patients who underwent pfHRP2 tests and 16,053 patients who underwent pLDH tests. The characteristics of each study are summarized in Table II. The flow chart of the literature search strategies is shown in Figure 1.

\section{Methodologic quality of included studies}

The quality of included studies was assessed using the QUADAS-2 tool, and the results are shown in Figure 2. Most studies enrolled a consecutive or random sample of eligible patients, but in one study the procedure of patient enrollment was not clearly described [20]. Three studies were considered to be inappropriate because the studies excluded severe or special patients (such as pregnant women) from the studies [20-22]. Seven studies did not supply sufficient information about the blindness of index or reference standard tests [4, 6-8, 16, 22, 23]. Four studies reported that the tests were not performed or the results were missed in a portion of the patients $[14,20$, $24,25]$. According to the criteria of the evaluation, $75 \%$ of studies had a low risk of bias in the patient selection domain, $50 \%$ of studies were judged to have low risk in the other 3 domains, and only 
A

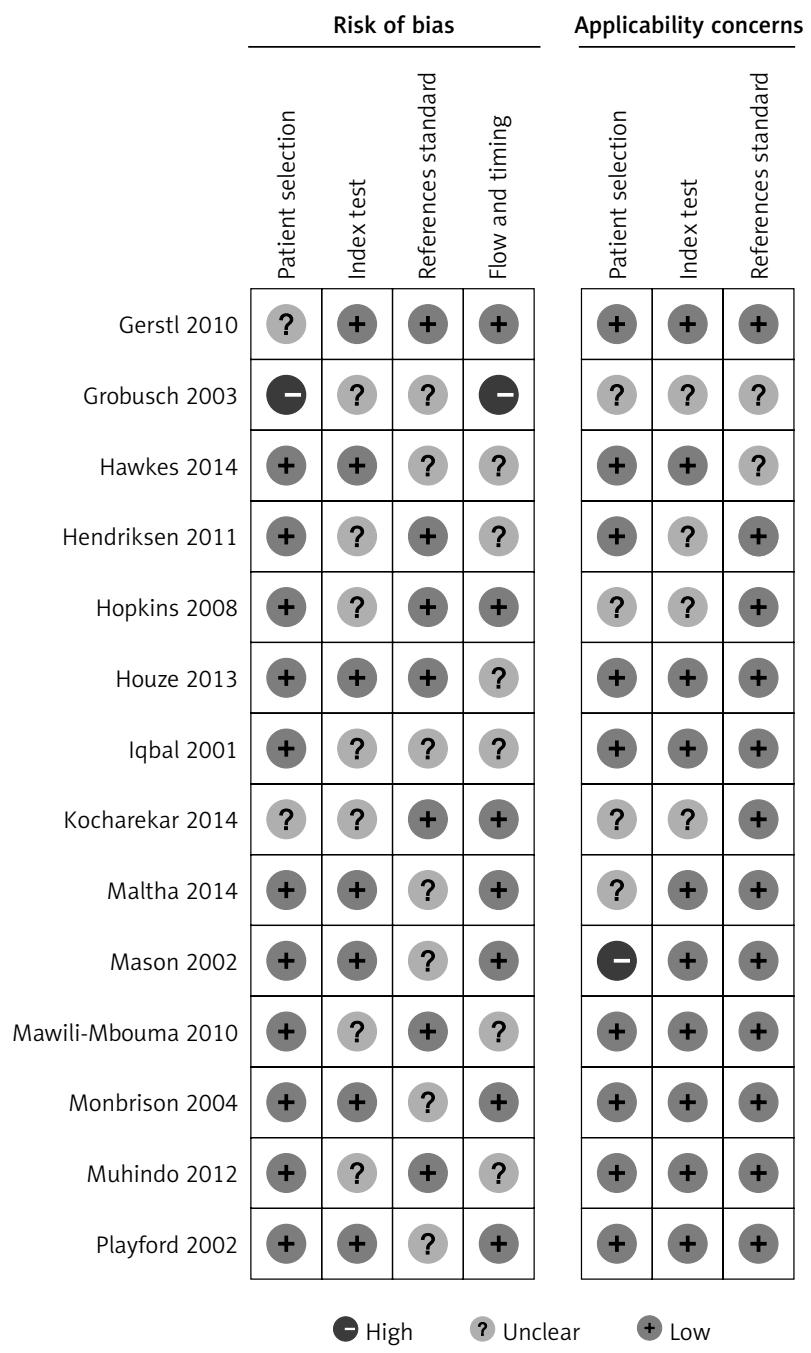

B

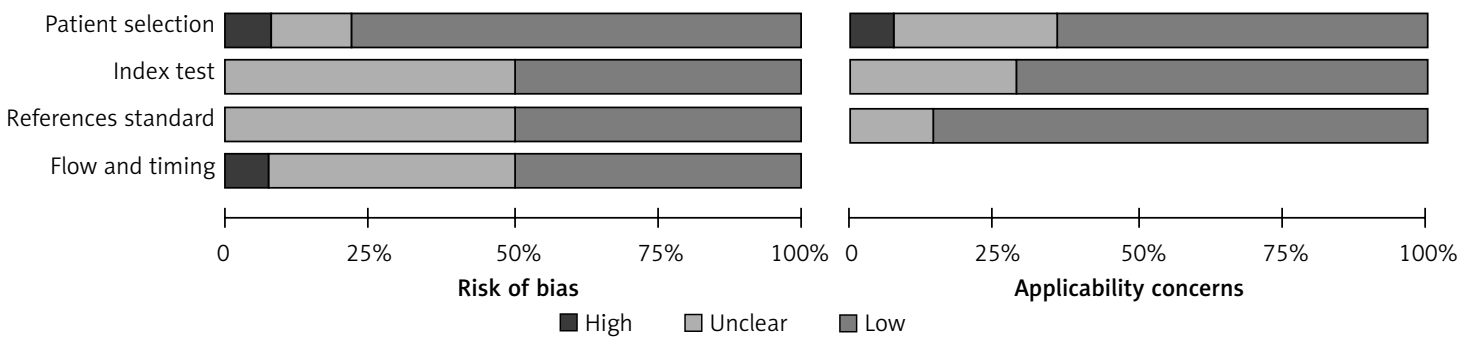

Figure 2. A - Risk of bias and applicability concerns summary: review authors' judgments about each domain of the QUADAS-2 checklist for each study. B - Risk of bias and applicability concerns graph: review authors' judgments about each domain presented as percentages across included studies

1 study was assessed to be at high risk of bias in patient selection and flow and timing domains [20]. In the applicability section, only 1 study was evaluated as high concern in the patient selection domain [15]. The remaining studies had low or unclear concerns.

\section{Heterogeneity analysis}

To determine whether or not there was heterogeneity in the studies, the Spearman correlation coefficient was used to assess the presence of a threshold effect. The results suggest that there was no threshold effect in the pfHRP2 ( $r s=0.332$, $p=0.246)$ and $\mathrm{pLDH}(r s=0.231, p=0.427)$ tests. The heterogeneity caused by other sources was analyzed by Cochrane's $Q$ test and the $\chi^{2}$ test. The results showed that there was clear heterogeneity in the pfHRP2 (DOR Cochrane $Q=357.77$, $p=0.000, l^{2}=96.4 \%$ ) and pLDH (DOR Cochrane $\left.Q=208.95, p<0.001, l^{2}=93.8 \%\right)$ tests. Meta-regression analysis was therefore used to determine 


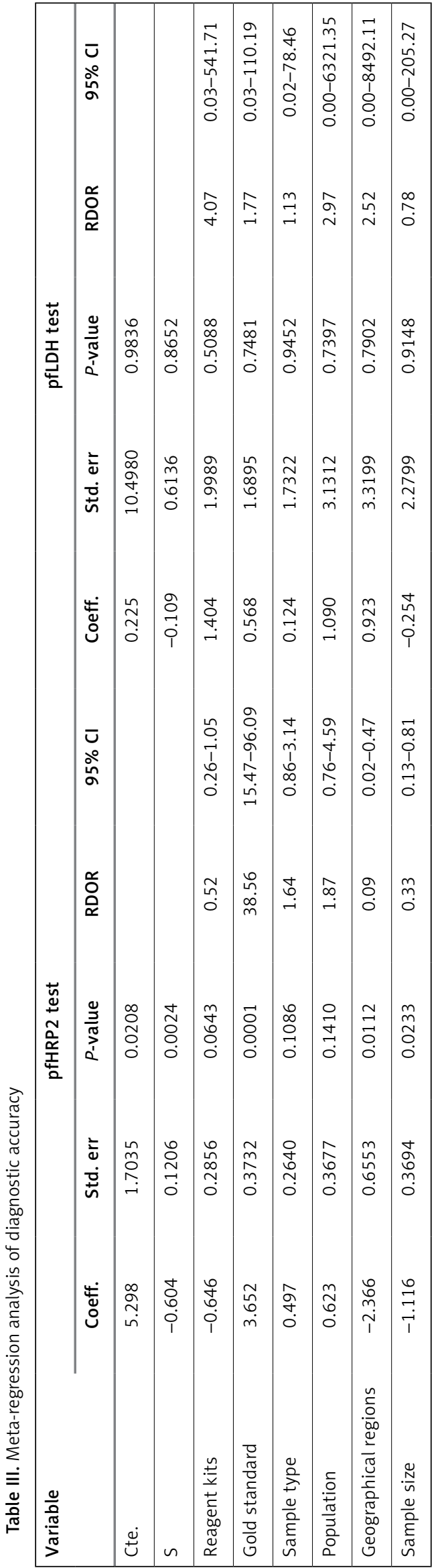

the sources of the heterogeneity subsequently. The results suggested that the selection of the gold standard test was the major source of heterogeneity in the pfHRP2 test, but there was no definite source of heterogeneity identified in the pLDH test. The results of the meta-regression are shown in Table III.

\section{Meta-analysis}

The random effects model was used to calculate the pooled value because of heterogeneity in the tests; the forest plots and ROCs are shown in Figures 3 and 4 . The results of meta-analysis demonstrated that the pooled sensitivity and specificity were $96.3 \%$ (95.8-96.7\%) and 86.1\% (85.3-86.8\%) in the pfHRP2 test and $82.6 \%(81.7-83.5 \%)$ and 95.9\% (95.4-96.3\%) in the pLDH test. The pooled ROCs showed an AUC of 0.982 in the pfHRP2 test and 0.985 in the pLDH test. The DOR was 243.31 in the pfHRP2 and 230.59 in the pLDH tests; the pooled data of the two tests are listed in Table IV.

\section{Subgroup analysis}

According to the gold standard test selected, 14 studies were classified into two groups for subgroup analysis, including PCR-corrected microscopy groups (6 studies) and pure microscopy groups (8 studies). Pooled sensitivity, specificity, PLR, NLR, and DOR for the two groups in the pfHRP2 test are presented in Table IV. Because there was no definite source of heterogeneity identified in the pLDH tests, subgroup analysis was not performed.

\section{Discussion}

Although similar studies were conducted by Marx et al. [26] and Abba et al. [27], a number of relevant studies have been published in the past 5 years. Therefore, we performed this meta-analysis to better clarify the role of pfHRP2- and pLDH-based RDTs in the diagnosis of $P$. falciparum infections. Unlike the previous meta-analyses, we only included studies which directly compared the diagnostic performance of pfHRP2- and pLDH-based tests to avoid the heterogeneity of population selection.

Fourteen studies which met the inclusion criteria were included in our study, including 15,909 patients who had pfHRP2 tests and 16,053 patients who had pLDH tests. The QUADAS- 2 tool was used to evaluate the included studies. The results indicated that most of the included studies were of high quality and considered to provide reliable results.

The present meta-analysis demonstrated that the pfHRP2 tests had a pooled sensitivity of $96.3 \%$ and a pooled specificity of $86.1 \%$ for detection of $P$. falciparum. The pLDH test reported an $82.6 \%$ pooled sensitivity and a $95.9 \%$ pooled specificity, 


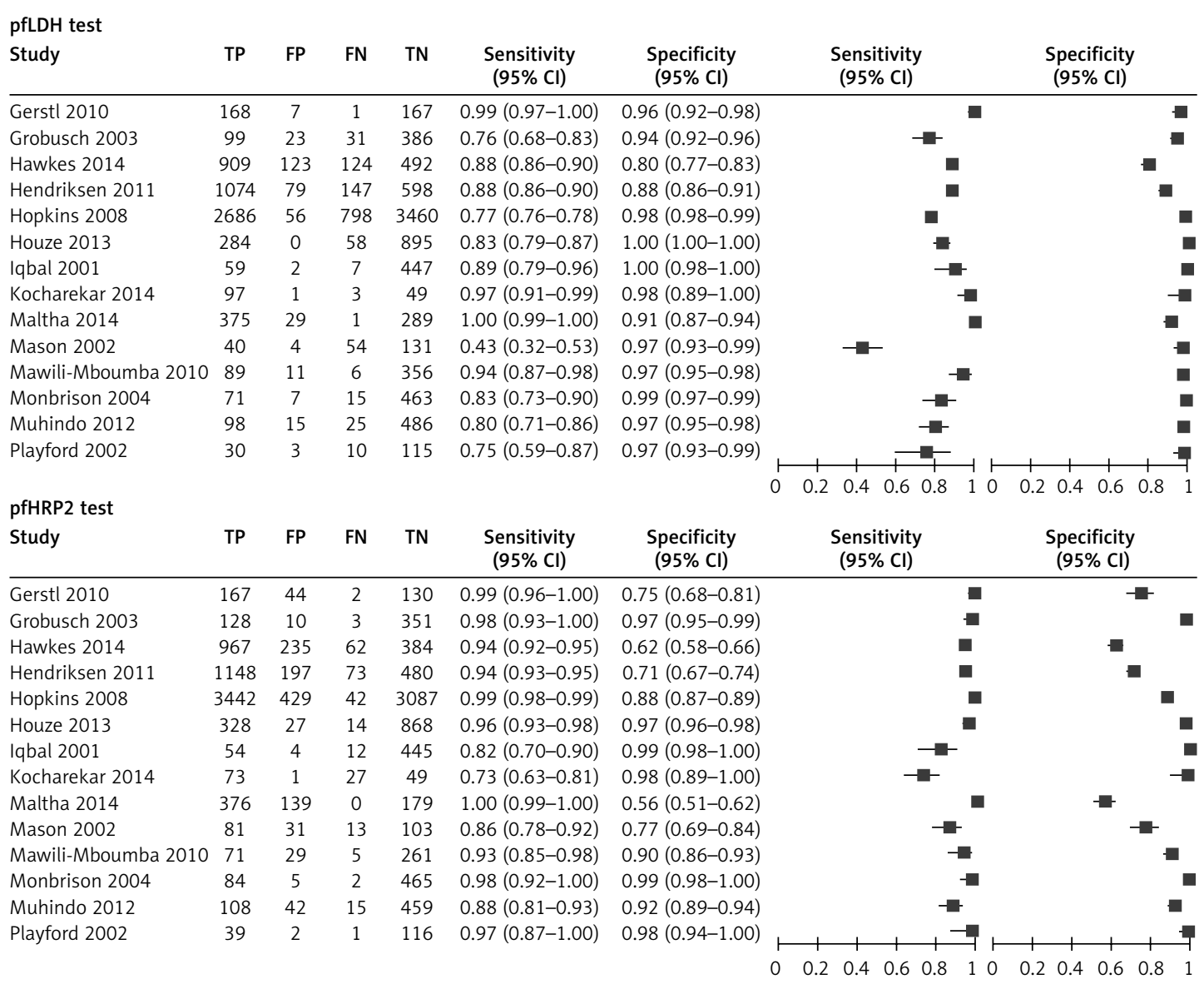

Figure 3. Forest plot of pairs of sensitivity and specificity in each study

which showed lower sensitivity and better specificity compared to the PfHRP2 test. Overall, these results are consistent with previously published studies, but there were slight decreases in sensitivity and specificity compared with the Abba et al. report [27]. The performance of RDTs is influenced by many factors, such as quality of reagent kits, parasite density, storage conditions and interpretation of the test results. Many studies have reported that genetic diversity and deletion could affect the specificity of pfHRP2 tests. However, the sensitivity of the PfHRP2 tests is higher than that of the pLDH test, perhaps due to the persistence of antigens [12, 13]. The pooled ROCs showed an AUC of 0.982 in the pfHRP2 tests and 0.985 in the pLDH tests, indicating promising discriminative abilities for both tests. The DOR, which estimates the odds of positive test results between diseased and non-diseased groups, was 243.31 for the pfHRP2 tests and 230.59 for the pLDH tests, indicating that the PfHRP2 tests had slightly greater accuracy compared to the pLDH tests.

The results of heterogeneity analysis demonstrated that there was no threshold effect for both RDTs, but obvious heterogeneities caused by other sources were found among these studies. Meta-regression analysis showed that selection

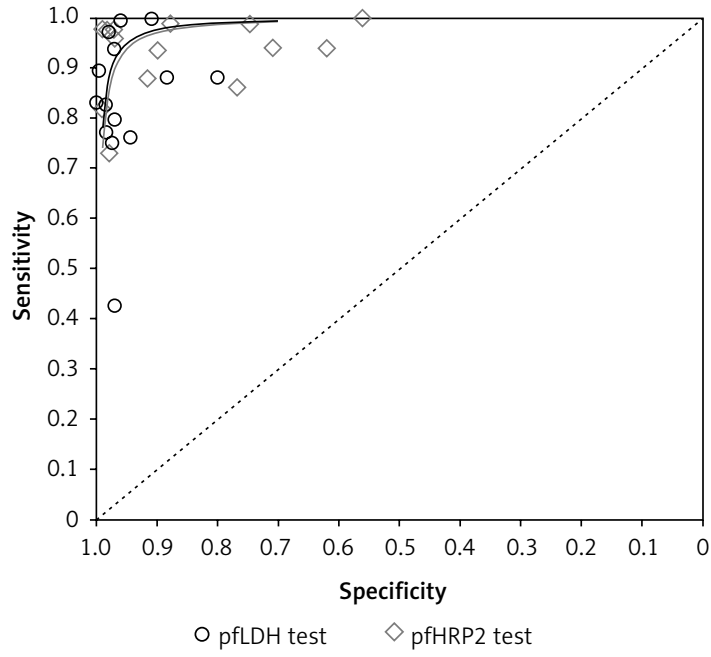

Figure 4. Summary receiver operating characteristic curves for pfHRP2- and pLDH-based tests

of a gold standard test affected the heterogeneity significantly in the PfHRP2 test $(\mathrm{RDOR}=38.56$, $p=0.0001$ ). To evaluate the influence of the selection of the gold standard test on the diagnostic performance of the test, subgroup analysis was conducted and the heterogeneity was significantly decreased when studies were divided into PCR-corrected (DOR Cochrane $Q=7.61, p=0.179$, 


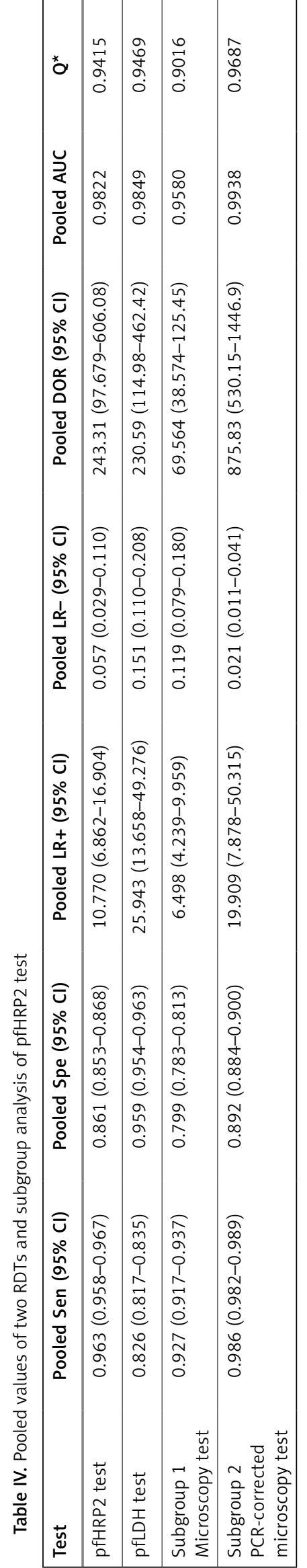

$\left.P^{2}=34.3 \%\right)$ and microscopy groups (DOR Cochrane $\left.Q=47.33, p<0.001, R^{2}=85.2 \%\right)$. A meta-analysis of the subgroup indicated that tests with PCR-corrected microscopy had higher sensitivity, specificity, DOR, and AUC (data are shown in Table IV) than tests with pure microscopy. However, meta-regression analysis showed that the selection of the gold standard test did not significantly affect heterogeneity in the pLDH tests. It can be explained by the prolonged persistence of HRP-2 antigen in the circulation. After successful anti-malarial treatment, pLDH tests and routine microscopy cannot reliably detect very low parasite densities, while PCR and the PfHRP2 test provide higher sensitivity at low densities than that of the other two methods. In addition, the performance of the RDTs has been reported to be variable in different regions [12]. In this meta-analysis, all included studies were divided into two categories: endemic areas and non-endemic areas. Results of meta-regression analysis showed that the heterogeneity of RDTs was slightly influenced by geographical origin of the samples (pfHRP2 test: RDOR $=0.09, p=$ 0.0112 ; pLDH test: $\mathrm{RDOR}=2.52, p=0.7902)$. Another possible factor affecting the performance of RDTs is the quality of the reagent kits, but we did not conduct a meta-analysis according to brands of reagents because the number of included studies was small. However, the meta-regression analysis showed that the heterogeneity cannot be affected by brands of reagents in two RDTs.

A number of limitations regarding the current study need to be considered. First, the diversity of samples could be a potential source of bias because the outcomes of RDTs may be influenced by the parasite densities in the blood samples. Therefore, we attempted to perform a subgroup analysis according to the parasite densities, but some essential data could not be obtained from the studies. Second, there was considerable heterogeneity in the pLDH tests, but we failed to find a reasonable source of the heterogeneity. Therefore, the results of pLDH tests should be interpreted cautiously.

In conclusion, our results suggest that the two rapid tests we evaluated performed satisfactorily for the diagnosis of $P$. falciparum, but the pLDH tests had higher specificity, while the pfHRP2 tests offered better sensitivity. In general, the pfHRP2 tests showed slightly greater accuracy compared to pLDH tests according to this meta-analysis. We conclude that a combination of both antigens might be a more reliable approach for the diagnosis of malaria.

\section{Acknowledgments}

Co-first authors: Bo Li and Zhiqiang Sun

Source of support: the National Major "Twelfth Five-Year Plan" project of science and technology (2013ZX09J13106-06A). 


\section{Conflict of interest}

The authors declare no conflict of interest.

\section{References}

1. WHO. World Malaria Report 2012. Geneva 2012.

2. Hopkins H, Kambale W, Kamya MR, Staedke SG, Dorsey G, Rosenthal PJ. Comparison of HRP2- and pLDHbased rapid diagnostic tests for malaria with longitudinal follow-up in Kampala, Uganda. Am J Trop Med Hyg 2007; 76: 1092-7.

3. Hopkins H, Bebell L, Kambale W, Dokomajilar C, Rosenthal PJ, Dorsey G. Rapid diagnostic tests for malaria at sites of varying transmission intensity in Uganda. J Infect Dis 2008; 197: 510-8.

4. Iqbal J, Hira PR, Sher A, Al-Enezi AE. Diagnosis of imported malaria by Plasmodium lactate dehydrogenase (pLDH) and histidine-rich protein 2 (PfHRP-2)-based immunocapture assays. Am J Trop Med Hyg 2001; 64: 20-3.

5. Kattenberg JH, Ochodo EA, Boer KR, Schallig HD, Mens PF, Leeflang MM. Systematic review and meta-analysis: rapid diagnostic tests versus placental histology, microscopy and PCR for malaria in pregnant women. Malar J 2011; 10: 321.

6. Maltha J, Guiraud I, Lompo P, et al. Accuracy of PfHRP2 versus Pf-pLDH antigen detection by malaria rapid diagnostic tests in hospitalized children in a seasonal hyperendemic malaria transmission area in Burkina Faso. Malar J 2014; 13: 20.

7. Muhindo HM, llombe G, Meya R, et al. Accuracy of malaria rapid diagnosis test Optimal-IT $\left.{ }^{\circledR}\right)$ in Kinshasa, the Democratic Republic of Congo. Malar J 2012; 11: 224

8. Playford EG, Walker J. Evaluation of the ICT Malaria P.f/ P.v and the OptiMal Rapid Diagnostic Tests for malaria in febrile returned travellers. J Clin Microbiol 2002; 40: 4166-71.

9. Heutmekers M, Gillet P, Cnops L, et al. Evaluation of the malaria rapid diagnostic test SDFK90: detection of both PfHRP2 and Pf-pLDH. Malar J 2012; 11: 359.

10. Maltha J, Gamboa D, Bendezu J, et al. Rapid diagnostic tests for malaria diagnosis in the Peruvian Amazon: impact of pfhrp2 gene deletions and cross-reactions. PLoS One 2012; 7: e43094.

11. Maltha J, Gillet P, Cnops L, et al. Evaluation of the rapid diagnostic test SDFK40 (Pf-pLDH/pan-pLDH) for the diagnosis of malaria in a non-endemic setting. Malar J 2011; $10: 7$.

12. Gamboa D, Ho MF, Bendezu J, et al. A large proportion of $P$. falciparum isolates in the Amazon region of Peru lack pfhrp2 and pfhrp3: implications for malaria rapid diagnostic tests. PLoS One 2010; 5: e8091.

13. Deme AB, Park DJ, Bei AK, et al. Analysis of pfhrp2 genetic diversity in Senegal and implications for use of rapid diagnostic tests. Malar J 2014; 13: 34.

14. Houzé S, Boutron I, Marmorat A, et al. Performance of rapid diagnostic tests for imported malaria in clinical practice: results of a national multicenter study. PLoS One 2013; 8: e75486.

15. Mason DP, Kawamoto F, Lin K, Laoboonchai A, Wongsrichanalai C. A comparison of two rapid field immunochromatographic tests to expert microscopy in the diagnosis of malaria. Acta Tropica 2002; 82: 51-9.

16. Mawili-Mboumba DP, Bouyou Akotet MK, Ngoungou EB, Kombila M. Evaluation of rapid diagnostic tests for malaria case management in Gabon. Diagn Microbiol Infect Dis 2010; 66: 162-8.
17. Minja DT, Schmiegelow C, Oesterholt M, et al. Reliability of rapid diagnostic tests in diagnosing pregnancy-associated malaria in north-eastern Tanzania. Malar J 2012; 11: 211 .

18. Whiting PF, Rutjes AW, Westwood ME, et al. QUADAS-2: a revised tool for the quality assessment of diagnostic accuracy studies. Ann Intern Med 2011; 155: 529-36.

19. Zhu J, Su X, Li G, et al. The incidence of acute myocardial infarction in relation to overweight and obesity: a meta-analysis. Arch Med Sci 2014; 10: 855-62.

20. Grobusch MP, Hanscheid T, Gobels K, et al. Comparison of three antigen detection tests for diagnosis and follow-up of falciparum malaria in travellers returning to Berlin, Germany. Parasitol Res 2003; 89: 354-7.

21. Gerstl S, Dunkley S, Mukhtar A, De Smet M, Baker S, Maikere J. Assessment of two malaria rapid diagnostic tests in children under five years of age, with follow-up of false-positive pLDH test results, in a hyperendemic falciparum malaria area, Sierra Leone. Malar J 2010; 9: 28.

22. Kocharekar MM, Sarkar SS, Dasgupta D. Comparative study of modified quantitative buffy coat and two rapid tests in comparison with peripheral blood smear in malaria diagnosis in mumbai, India. J Parasitol Res 2014; 2014: 194651.

23. De Monbrison F, Gerome P, Chaulet JF, Wallon M, Picot S, Peyron F. Comparative diagnostic performance of two commercial rapid tests for malaria in a non-endemic area. Eur J Clin Microbiol Infect Dis 2004; 23: 784-6.

24. Hawkes M, Conroy AL, Opoka RO, et al. Use of a threeband HRP2/pLDH combination rapid diagnostic test increases diagnostic specificity for falciparum malaria in Ugandan children. Malar J 2014; 13: 43.

25. Hendriksen IC, Mtove G, Pedro AJ, et al. Evaluation of a PfHRP2 and a pLDH-based rapid diagnostic test for the diagnosis of severe malaria in 2 populations of African children. Clin Infect Dis 2011; 52: 1100-7.

26. Marx A, Pewsner D, Egger M, et al. Meta-analysis: accuracy of rapid tests for malaria in travelers returning from endemic areas. Ann Intern Med 2005; 142: 836-46.

27. Abba K, Deeks JJ, Olliaro PL, et al. Rapid diagnostic tests for diagnosing uncomplicated P. falciparum malaria in endemic countries. Cochrane Database Syst Rev 2011; 7: CD008122. 